\title{
Predictors of Suicide Attempts in Clinically Depressed Korean Adolescents
}

\author{
Ahye Kwon ${ }^{1}$, Jungeun Song ${ }^{2}$, Ki-Hwan Yook ${ }^{3}$, Duk-In Jon ${ }^{1}$, Myung Hun Jung ${ }^{1}$, Narei Hong ${ }^{1}$, Hyun Ju Hong ${ }^{1,4}$ \\ ${ }^{1}$ Department of Psychiatry, Hallym University Sacred Heart Hospital, Hallym University College of Medicine, Anyang, ${ }^{2}$ Department of \\ Psychiatry, National Health Insurance Service IIsan Hospital, Goyang, ${ }^{3}$ Department of Psychiatry, CHA Bundang Medical Center, CHA \\ University, Seongnam, ${ }^{4}$ Suicide and School Mental Health Institute, Hallym University, Anyang, Korea
}

\begin{abstract}
We examined predictors of suicide attempts in clinically depressed adolescents in Korea and gender differences in suicidal behavior. In total, 106 adolescents diagnosed with depressive disorder were recruited in South Korea. We assessed various variables that might affect suicide attempts, and used a structured interview for the diagnosis of depression and comorbidities and to evaluate suicidality. Demographic and clinical characteristics of the subjects were compared between suicide attempt and non-suicide attempt groups and we examined significant predictors of suicide attempts. Gender differences in suicidal ideation and suicidal behavior were also analyzed. Among 106 depressed participants, 50 (47.2\%) adolescents were classified in the suicide attempt group. Generally, the suicide attempt and non-suicide attempt group shared similar clinical characteristics. The suicide attempt group had more females, more major depressive disorder diagnoses, more depressive episodes, and higher suicidal ideation than the non-suicide attempt group. Suicidal ideation was the only significant predictor of suicidal attempt, regardless of gender. Higher suicidal ideation frequency scores and more non-suicidal self-injurious behaviors were shown in the female suicide attempt group than the male suicide attempt group. It is recommended that suicidal ideation be assessed regularly and managed rigorously to decrease suicide risks in depressive adolescents.
\end{abstract}

KEY WORDS: Adolescent; Depression; Suicide; Suicide, attempted; Suicidal ideation.

\section{INTRODUCTION}

Suicide is a major public health problem in many countries. $^{1-3)}$ The mean suicide rate in the 15-19 years of age group was 7.4/100,000 in 90 of the 130 World Health organization member states. ${ }^{4)}$ Previous suicide attempts and major depression are major predictors of completed suicide in adolescents. ${ }^{5} \mathrm{Up}$ to $60 \%$ of adolescent suicide victims had a depressive disorder at the time of death ${ }^{6}$ but not all depressive adolescents show suicidal behavior. About $60-70 \%$ of depressed youth have suicidal ideation or thoughts, and $13-39 \%$ have attempted suicide. ${ }^{7,8)}$

Few studies have examined the clinical characteristics of those who have suicidality in a depressed youth sample. Depressed suicidal children and adolescents had greater depression severity, more comorbid conditions, poorer functional status, more hopelessness, more insomnia,

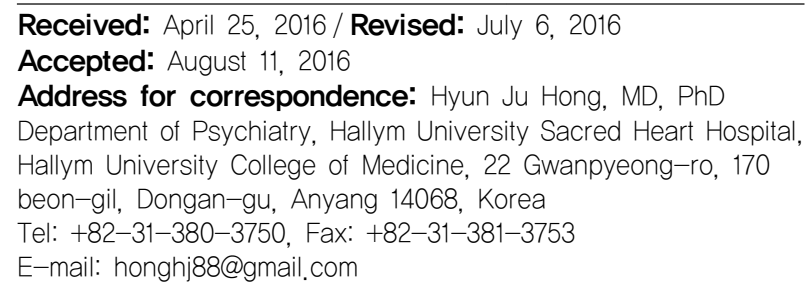

more non-suicidal self-injury and poorer family function compared with the non-suicidal group. ${ }^{9-12)}$ However the assessment of suicidality was dependent on one or two questions, definitions of suicidality were inconsistent, and most did not differentiate suicidal attempt and ideation. ${ }^{8-11)}$

The purposes of our study were to examine predictors of suicide attempts in clinically depressed adolescents in Korea, and gender difference in suicidal ideation and attempts. We adopted a clearer definition of suicide attempt by using a standardized structured interview and a more accurate diagnosis of depression using a structured diagnostic interview and the regular diagnostic meeting. We assessed a broad range of variables that might affect suicidal behavior.

\section{METHODS}

\section{Subjects}

The subjects were 13-18 years of age and had visited the psychiatric outpatient clinics of three university hospitals in South Korea (Hallum University Sacred Heart Hospital, Kwandong University Myongji Hospital, CHA Bundang Medical Center) from December 2010 through August

(a) This is an Open-Access article distributed under the terms of the Creative Commons Attribution Non-Commercial License (http://creativecommons.org/licenses/by-nc/4.0) which permits unrestricted non-commercial use, distribution, and reproduction in any medium, provided the original work is properly cited. 
2013. Subjects who met the Diagnostic and Statistical Manual of Mental Disorders 4th edition (DSM-IV) criteria for depressive disorders (major depressive disorder, dysthymic disorder, or depressive disorder not otherwise specified) were included. Exclusion criteria included a lifetime diagnosis of a psychotic disorder, bipolar disorder, autism, mental retardation, any mental disorder due to general medical condition, any brain disease or epilepsy. In total, 106 adolescents were enrolled and completed a battery of self-reported questionnaires, their parents also completed questionnaires.

This study was approved by the Institutional Review Board of the ethical committee at each hospital (IRB No. 2010-I084). Written informed consents were obtained.

\section{Diagnosis and Clinical Assessment}

Diagnoses of depression and comorbidities were made by well-trained psychiatrists or master's degree level research assistants using the Kiddle-Schedule for Affective Disorder and Schizophrenia Present and Lifetime Korean Version (K-SADS-PL-K) ${ }^{13)}$ The final diagnosis was confirmed after discussion with the research team in regular diagnostic meetings.

We used the Columbia Suicide Severity Rating Scale (C-SSRS $)^{14,15)}$ to evaluate suicidal ideation and attempts. First, the severity of ideation is measured, rated on a five-point ordinal scale. Second, the intensity of the suicidal ideation subscale consists of five items, each rated on a five-point ordinal scale: frequency, duration, controllability, deterrents, and reason for ideation. The third is a behavior subscale, which includes actual, aborted, and interrupted attempts and non-suicidal self-injurious behavior. The fourth is a lethality subscale, which assessed actual suicide attempts' lethality rated on a six-point ordinal scale.

Subjects with actual, aborted, and interrupted suicide attempts were classified as the 'suicide attempt' group. The 'non-suicide attempt' group included those having no suicidal ideation, non-suicidal self-injurious behavior, and suicidal ideation without any suicide behavior.

Age, gender, family structure, and major stress factors were evaluated. Physical abuse was assessed by a modified and translated version ${ }^{16)}$ of the violence scale in the Conflict Tactics Scale. ${ }^{17)}$ Both bullying perpetrators and victimization were assessed with the Korean version of the Bullying Behavior Scale ${ }^{18,19)}$ and the Peer-victimization Scale. ${ }^{19,20)}$ We used the resilience scale ${ }^{21)}$ which defines resilience as an ability to overcome the faced difficulties and adapt to new circumstances so as to enable spiritual growth.

\section{Statistical Analyses}

Demographic and other clinical characteristics of the subjects were compared between suicide attempt and non-suicide attempt groups using $\chi^{2}$ tests (for categorical variables) and independent-sample $t$-tests (for continuous variables). Multiple logistic regression analysis was used to determine which variables were independent predicting factors for suicide attempts. Variables that showed relative associations with suicide attempts in univariate analyses $(p<0.1)$ were entered into this regression analysis. To examine gender differences between the suicide attempt and non-suicide groups, the procedures above were performed for female and male groups. Finally, based on the C-SSRS items, gender differences in suicidal ideation and suicidal behavior patterns in the suicide attempt group were assessed. All tests were considered significant at $p=0.05$ (2-tailed). Data were analyzed using SPSS ver. 17.0 (SPSS Inc, Chicago, IL, USA).

\section{RESULTS}

\section{Clinical Characteristics of the Suicide Attempt and Non-suicide Attempt Groups}

Among 106 depressed participants, 50 (47.2\%) adolescents were classified into the suicide attempt group. Demographic and clinical characteristics of the suicide attempt and non-suicide attempt groups are shown in Table 1. There were significant differences in gender, prevalence of major depressive disorder (MDD) diagnosis, number of MDD episodes, and suicidal ideation between the groups. Male gender, MDD diagnosis, number of MDD episodes, and suicidal ideation were significant predictors in the univariate analyses, but suicidal ideation was the only significant predictor in the multivariate analysis (Table 2). When we checked multicollinearity using SPSS between independent variables, the variance inflation factors were 1.1-1.3, indicating no significant multicollinearity.

When these analyses were repeated in the female group, there were significant differences in the prevalence of MDD diagnosis, previous number of MDD episodes and bullying victimization between the groups. MDD diagnosis (odds ratio $[\mathrm{OR}]=4.17,95 \%$ confidence interval $[\mathrm{CI}]=1.27-13.65)$, number of MDD episodes $(\mathrm{OR}=2.53$, $95 \% \mathrm{CI}=1.09-5.86)$, and suicidal ideation $(\mathrm{OR}=2.59,95 \%$ $\mathrm{CI}=1.63-4.10)$ were significant predictors in univariate analyses, but suicidal ideation $(\mathrm{OR}=2.51,95 \% \mathrm{CI}=1.37-4.59)$ was the only significant risk factor in the multivariate analysis. When these analyses were performed in the male group, there were no significant differences between the 
Table 1. Demographic and clinical characteristics of suicide attempt and non-suicide attempt groups

\begin{tabular}{|c|c|c|c|c|}
\hline Variable & Suicide attempt $(n=50)$ & Non-suicide attempt $(n=56)$ & $t / \chi^{2}$ & $p$ value \\
\hline Age $(y r)$ & $15.6 \pm 1.8$ & $15.5 \pm 1.7$ & -0.446 & 0.657 \\
\hline \multicolumn{5}{|l|}{ Gender } \\
\hline Male & $11(31.4)$ & $24(68.6)$ & \multirow[t]{2}{*}{5.196} & \multirow[t]{2}{*}{$0.023^{*}$} \\
\hline Female & $39(54.9)$ & $32(45.1)$ & & \\
\hline \multicolumn{5}{|l|}{ Stress factor } \\
\hline Academic stress & $14(28.6)$ & $22(41.5)$ & 1.866 & 0.172 \\
\hline Peer relational stress & $13(26.5)$ & $20(37.7)$ & 1.461 & 0.227 \\
\hline Family related stress & $16(32.7)$ & $10(18.9)$ & 2.547 & 0.110 \\
\hline Others & $15(30.6)$ & $9(16.7)$ & 2.796 & 0.095 \\
\hline \multicolumn{5}{|l|}{ Family structure } \\
\hline Living with both parents & $27(61.4)$ & $33(67.3)$ & \multirow[t]{2}{*}{0.363} & \multirow[t]{2}{*}{0.547} \\
\hline Others $^{\dagger}$ & $17(38.6)$ & $16(32.7)$ & & \\
\hline \multicolumn{5}{|l|}{ Diagnosis } \\
\hline MDD & $38(77.6)$ & $30(57.7)$ & \multirow[t]{2}{*}{4.523} & \multirow[t]{2}{*}{$0.033^{*}$} \\
\hline Others $^{\ddagger}$ & $11(22.4)$ & $22(42.3)$ & & \\
\hline Number of MDD episodes & $1.4 \pm 1.2$ & $0.8 \pm 0.5$ & -3.175 & $0.002^{*}$ \\
\hline Number of comorbidities & $0.6 \pm 0.7$ & $0.7 \pm 0.9$ & 0.502 & 0.617 \\
\hline First depressive episode onset age & $13.9 \pm 2.4$ & $13.7 \pm 1.9$ & -0.381 & 0.704 \\
\hline Physical abuse & $11.1 \pm 11.2$ & $7.4 \pm 8.5$ & -1.953 & 0.054 \\
\hline Bullying & $19(42.2)$ & $26(57.8)$ & 0.756 & 0.385 \\
\hline Perpetrator & $5.4 \pm 3.5$ & $5.2 \pm 3.1$ & -0.313 & 0.755 \\
\hline Victimization & $4.8 \pm 4.2$ & $3.8 \pm 2.6$ & -1.341 & 0.184 \\
\hline Resilience & $76.2 \pm 15.1$ & $80.5 \pm 12.5$ & 1.602 & 0.112 \\
\hline Suicidal ideation severity of C-SSRS & $4.3 \pm 1.2$ & $2.2 \pm 1.4$ & -8.460 & $0.000^{*}$ \\
\hline
\end{tabular}

Values are presented as mean \pm standard deviation or number (\%).

MDD, major depressive disorder; C-SSRS, Columbia Suicide Severity Rating Scale.

${ }^{*} p<0.05$

${ }^{\dagger}$ Only one parent (bereaved, divorced, separated), a parent and a step-parent, grandparents raised family and others; ${ }^{\dagger}$ dysthymia, depressive disorder not otherwise specified.

Table 2. Logistic regression analysis of demographic and psychological variables for the suicide attempt group

\begin{tabular}{|c|c|c|c|c|c|c|}
\hline \multirow{2}{*}{ Variable } & \multicolumn{3}{|c|}{ Unadjusted } & \multicolumn{3}{|c|}{ Adjusted* } \\
\hline & B & OR & $95 \% \mathrm{Cl}$ & B & OR & $95 \% \mathrm{Cl}$ \\
\hline Gender & -0.978 & 0.376 & $0.160-0.883^{\dagger}$ & -0.359 & 0.699 & $0.200-2.442$ \\
\hline Diagnosis (MDD and others) & 0.930 & 2.533 & $1.064-6.033^{\dagger}$ & -1.224 & 0.294 & $0.061-1.419$ \\
\hline Number of MDD episodes & 0.973 & 2.647 & $1.336-5.245^{\ddagger}$ & 0.611 & 1.842 & $0.779-4.353$ \\
\hline Suicidal ideation severity of C-SSRS & 1.158 & 3.182 & $2.085-4.857^{\ddagger}$ & 1.253 & 3.499 & $2.063-5.936^{\ddagger}$ \\
\hline
\end{tabular}

B, beta coefficient; OR, odds ratio; Cl, confidence interval; MDD, major depressive disorder; C-SSRS, Columbia Suicide Severity Rating Scale. ${ }^{*}$ Adjusted for all other variables in the model; ${ }^{\dagger} p<0.05,{ }^{\dagger} p<0.01$.

groups except for suicidal ideation. Suicidal ideation $(\mathrm{OR}=6.24,95 \% \mathrm{CI}=1.81-21.47)$ was the only significant risk factor in the regression analysis.

\section{Gender Differences of Suicidal Ideation and Suicide Behaviors in Suicide Attempt Group}

The suicidal ideation frequency score of C-SSRS was higher in girls (mean, 3.8; standard deviation [SD], 1.3) than boys (mean, 2.6; SD, 1.8). Moreover, girls $(n=27$, $73.0 \%$ ) who attempted suicide reported more non-suicidal self-injurious behaviors than boys $(n=2,20.0 \%)$ who attempted suicide. Other items showed no significant gen- der difference in the suicide attempt group.

\section{DISCUSSION}

This study explored the clinical characteristics of a suicide attempt group and predictors of suicide attempt in clinically depressed Korean adolescents.

Generally, the suicide attempt and non-suicide attempt groups shared similar clinical characteristics. Several demographic and clinical features, including family structure and physical abuse, showed no significant difference between the two groups. Although the suicide attempt 
group had more females, more MDD diagnoses, more depressive episodes, and higher suicidal ideation than the non-suicide attempt group, suicidal ideation was the only significant predictor of suicide attempts regardless of gender. This result suggests that the suicide attempt group in adolescent depression might not be a distinctive subgroup of depressive adolescents and supports previous studies that reported little difference in clinical features between the two groups. ${ }^{10,22)}$ All of our subjects in our study had significant depressive symptoms, and even the non-suicide attempt group might have had experiences of non-suicidal self-mutilation or have had serious suicidal ideation without any suicidal behavior. This means that the non-suicide attempt group may have a higher suicide risk than the general population, and that there is little difference between the suicide attempt and non-suicide attempt groups in depressive adolescents.

Several studies have reported significant clinical differences between suicidal and non- suicidal groups in depressed adolescents. ${ }^{8-10)}$ Differences between our study and those studies might be related to differences in the definition of suicidality or the range of independent variables or subject characteristics. In previous studies, the suicidal group included subjects with suicidal ideation, and suicidal ideation was not an independent predictor of suicidal behavior. Our study adopted a clear definition of suicide attempt that excluded suicidal ideation and assessed a broad range of variables as independent variables, including suicidal ideation. Our sample might also represent severe depressive adolescents because tertiary university hospital patients were enrolled.

Repetitive, intensive, continuous suicidal ideation is significant predictor of planned suicide attempt and the suicide ideation tends to rapidly progress to suicidal behavior in planned suicide attempters. ${ }^{23)}$ Suicidal ideation is a well-known predictor of suicide attempts in community adolescent samples as well as adults. ${ }^{24-27)}$ In a Norwegian study, the strongest predictor of previous suicide attempts was suicidal ideation. In an initial univariate analysis, depressive mood was a significant predictor but its effect was reduced when suicidal ideation was controlled. ${ }^{24)}$ This is similar to our result about the relationship between suicidal ideation and depression in suicide attempts.

The developmental trajectories of suicidal ideation and attempts in children and adolescents differ by age and gender. Suicidal ideation and attempts are more prevalent in adolescents than children. ${ }^{10)}$ and the average age of onset occurs between 10 and 15 years of age. ${ }^{28)}$ The prevalence of suicidal ideation increases rapidly between the ages of 12 and 17 while the rates of plans and attempts rise increasingly between the ages of 12 and 15 and then more slowly until age 17 in a United States national sample study. One-third (33.4\%) of suicide ideators develop a suicide plan and $33.9 \%$ make an attempt. ${ }^{29)}$

In our study, $47.2 \%$ of the adolescents were classified into the suicide attempt group. This is greater percentage than that reported by previous studies. ${ }^{78}$ Our subjects were recruited from tertiary university hospitals and the definition of the suicide attempt group differed from that in the previous studies. The suicide attempt group included aborted and interrupted attempts as well as actual suicide attempters according to C-SSRS. If the suicide attempt group had included only actual suicide attempt, the prevalence would be $37.7 \%$ which is similar to the other studies.

Another interesting point in our study is the gender difference in suicidal behavior in depressive adolescents. Although the number of MDD episodes and suicidal ideation were significant predictors of suicide attempt in the univariate analyses in the female group, suicidal ideation was the only significant predictor in the male group. The female suicidal attempt group had higher suicidal ideation frequency scores and more non-suicidal self-injurious behaviors than the male. This suggests a gender-specific strategy for decreasing suicide risks.

Although the small number of participants and crosssectional analysis raise the problem of generalizability and difficulty in showing a causal relationship, our study shows that suicidal ideation is the only and strongest predictor of suicide attempts in depressed adolescents. To decrease suicide risk in depressive adolescents, it may be useful to assess suicidal ideation regularly and manage it rigorously because suicidal ideation can be viewed as the gateway to serious suicidal acts.

\section{acknowledgments}

This research was supported by Hallym University Research Fund, 01-2011-11.

\section{REFERENCES}

1. Statistics Korea. Cause of death statistics: Statistics Korea; 2012 [Internet]. Daejeon: Statistics Korea; 2012 [cited at $2016 \mathrm{Feb}$ 17]. Available from: http://kostat.go.kr/portal/ korea/kor_nw/2/6/1/index.board?bmode =read\&aSeq $=308559$.

2. Statistics Korea. Cause of death statistics: Statistics Korea; 2013 [Internet]. Daejeon: Statistics Korea; 2013 [cited at $2016 \mathrm{Feb}$ 17]. Available from: http://kostat.go.kr/portal/ korea/kor ko/5/2/index.board?bmode $=$ read\&aSeq $=330389$.

3. Statistics Korea. Cause of death statistics: Statistics Korea; 2014 [Internet]. Daejeon: Statistics Korea; 2014 [cited at $2016 \mathrm{Feb}$ 17]. Available from: http://kostat.go.kr/portal/ 
korea/kor_ko/5/2/index.board?bmode $=$ read\&aSeq $=348646$.

4. Wasserman D, Cheng Q, Jiang GX. Global suicide rates among young people aged 15-19. World Psychiatry 2005;4:114-120.

5. American Academy of Child and Adolescent Psychiatry. Practice parameter for the assessment and treatment of children and adolescents with suicidal behavior. American Academy of Child and Adolescent Psychiatry. J Am Acad Child Adolesc Psychiatry 2001;40(7 Suppl):24S-51S.

6. Brent DA, Baugher M, Bridge J, Chen T, Chiappetta L. Ageand sex-related risk factors for adolescent suicide. J Am Acad Child Adolesc Psychiatry 1999;38:1497-1505.

7. Kovacs M, Goldston D, Gatsonis C. Suicidal behaviors and childhood-onset depressive disorders: a longitudinal investigation. J Am Acad Child Adolesc Psychiatry 1993;32:8-20.

8. Yorbik O, Birmaher B, Axelson D, Williamson DE, Ryan ND. Clinical characteristics of depressive symptoms in children and adolescents with major depressive disorder. $J$ Clin Psychiatry 2004;65:1654-1659.

9. Barbe RP, Williamson DE, Bridge JA, Birmaher B, Dahl $\mathrm{RE}$, Axelson DA, et al. Clinical differences between suicidal and nonsuicidal depressed children and adolescents. J Clin Psychiatry 2005;66:492-498.

10. Liu X, Gentzler AL, Tepper P, Kiss E, Kothencné VO, Tamás Z, et al. Clinical features of depressed children and adolescents with various forms of suicidality. J Clin Psychiatry 2006;67:1442-1450.

11. Tuisku V, Kiviruusu O, Pelkonen M, Karlsson L, Strandholm T, Marttunen M. Depressed adolescents as young adults - predictors of suicide attempt and non-suicidal self-injury during an 8-year follow-up. J Affect Disord 2014; 152-154:313-319.

12. Asarnow JR, Porta G, Spirito A, Emslie G, Clarke G, Wagner $\mathrm{KD}$, et al. Suicide attempts and nonsuicidal self-injury in the treatment of resistant depression in adolescents: findings from the TORDIA study. J Am Acad Child Adolesc Psychiatry 2011;50:772-781.

13. Kim YS, Cheon KA, Kim BN, Chang SA, Yoo HJ, Kim $\mathrm{JW}$, et al. The reliability and validity of kiddie-schedule for affective disorders and schizophrenia-present and lifetime version- Korean version (K-SADS-PL-K). Yonsei Med $J$ 2004:45:81-89.

14. Lee CS. A study of the reliability and validity of the Columbia-Suicide Severity Rating Scale in Korea. Gimhae: Inje University;2011. [Dissertation].

15. Posner K, Brown GK, Stanley B, Brent DA, Yershova KV, Oquendo MA, et al. The Columbia-Suicide Severity Rating Scale: initial validity and internal consistency findings from three multisite studies with adolescents and adults. Am J Psychiatry 2011;168:1266-1277.
16. Kwon JY, Ahn DH, Lee JS. The effect of physical abuse to the emotions and behavior in children. Korean J Child Adolesc Psychiatry 1992;3:73-83.

17. Straus MA. Measuring intrafamily conflict and violence: the Conflict Tactics (CT) Scales. J Marriage Fam 1979:41: 75-88.

18. Neary A, Joseph S. Peer victimization and its relationship to self-concept and depression among schoolgirls. Pers Individ Dif 1994;16:183-186.

19. Lee CJ, Kwak KJ. [Self-concept and social support according to bullying types]. Korean J Dev Psychol 2000;13:65-80. Korean.

20. Callaghan S, Joseph S. Self-concept and peer victimization among schoolchildren. Personal Individ Differ 1995;18:161163.

21. Shin WY, Kim MG, Kim JH. Developing measures of resilience for Korean adolescents and testing cross, convergent, and discriminant validity. Korean J Youth Stud 2009;20:105-131.

22. Kosky R, Silburn S, Zubrick S. Symptomatic depression and suicidal ideation. A comparative study with 628 children. $J$ Nerv Ment Dis 1986;174:523-528.

23. Kim J, Lee KS, Kim DJ, Hong SC, Choi KH, Oh Y, et al. Characteristic risk factors associated with planned versus impulsive suicide attempters. Clin Psychopharmacol Neurosci 2015; 13:308-315.

24. Wichstrøm L. Predictors of adolescent suicide attempts: a nationally representative longitudinal study of Norwegian adolescents. J Am Acad Child Adolesc Psychiatry 2000;39: 603-610.

25. Lewinsohn PM, Rohde P, Seeley JR. Psychosocial risk factors for future adolescent suicide attempts. J Consult Clin Psychol 1994;62:297-305.

26. Reifman A, Windle M. Adolescent suicidal behaviors as a function of depression, hopelessness, alcohol use, and social support: a longitudinal investigation. Am J Community Psychol 1995;23:329-354.

27. Mendez-Bustos P, de Leon-Martinez V, Miret $M$, Baca-Garcia E, Lopez-Castroman J. Suicide reattempters: a systematic review. Harv Rev Psychiatry 2013;21:281-295.

28. Fergusson DM, Woodward LJ, Horwood LJ. Risk factors and life processes associated with the onset of suicidal behaviour during adolescence and early adulthood. Psychol Med 2000;30:23-39.

29. Nock MK, Green JG, Hwang I, McLaughlin KA, Sampson NA, Zaslavsky AM, et al. Prevalence, correlates, and treatment of lifetime suicidal behavior among adolescents: results from the National Comorbidity Survey Replication Adolescent Supplement. JAMA Psychiatry 2013;70:300-310. 